\title{
Calming the Electrical Storm: Use of Stellate Ganglion Block and Thoracic Epidural in Intractable Ventricular Tachycardia
}

\author{
Supriya D'souza, Shalini Saksena, Manju Butani \\ Department of Anaesthesiology, P D Hinduja Hospital and Medical Research Centre, Mumbai, Maharashtra, India
}

\section{Abstract}

Imbalances in the autonomic nervous system contribute to ventricular tachyarrhythmias. Sympatholysis with thoracic epidural analgesia or a stellate ganglion block attenuates myocardial excitability and the proarrhythmic effects of sympathetic hyperactivity.

Keywords: Stellate ganglion block, thoracic epidural analgesia, ventricular arrhythmias

\section{INTRODUCTION}

The term "electrical storm" (ES) describes a state of electrical instability of the heart characterized by clustering of recurrent episodes of ventricular tachycardia (VT) or ventricular fibrillation in a short period of time. We report a case where stellate ganglion block (SGB) and thoracic epidural analgesia (TEA) were used to manage this sympathetic storm.

\section{Case Report}

A 67-year-old hypertensive and diabetic male weighing 60-kg status postcoronary artery bypass graft surgery to left anterior descending and left circumflex arteries 4 years ago, presented to our institute with a history of chest pain, palpitations, and dyspnea since the last 30 days. Electrocardiogram revealed monomorphic VT during one such episode with a pulse of 170/ min and a blood pressure of $110 / 70 \mathrm{mmHg}$. He was started on injection Amiodarone $150 \mathrm{mg}$ intravenous (IV) bolus over $10 \mathrm{~min}$ followed by infusion of $1 \mathrm{mg} / \mathrm{min}$ IV for $6 \mathrm{~h}$ then $0.5 \mathrm{mg} / \mathrm{min}$ IV for $18 \mathrm{~h}$. The arrhythmia did not revert with this. Injection Metoprolol $5 \mathrm{mg}$, injection Lignocaine $100 \mathrm{mg}$, and injection Magnesium sulfate $2 \mathrm{~g}$ IV were tried but to no avail. Transthoracic echocardiography (TTE) revealed hypokinesia of apex, anterior, posterior, and inferior walls and septum with a reduced ejection fraction of $25 \%$. No clots were seen during this examination. No previous TTE report was available for comparison. Cardiac enzyme assay was normal and ruled out

\begin{tabular}{|l|l|}
\hline \multicolumn{3}{c|}{ Access this article online } \\
\hline Quick Response Code: & Website: \\
& www.ijccm.org \\
& \\
\end{tabular}

any active ischemia. He had stopped consuming his routine antiplatelet medications since the last fortnight.

Hypokalemia, hypomagnesemia, and hyperthyroidism were excluded as triggering factors for arrhythmias on laboratory investigations. He was subsequently given Direct current (DC) synchronized cardioversion with $100 \mathrm{~J}$, and normal sinus rhythm (NSR) was achieved. He went into monomorphic VT 8 times on day 1 [Figure 1] and was reverted only with increasing dose of synchronized cardioversion each time. Decision was made to try sympatholysis with a left-sided SGB (LSGB) on day 2. After checking coagulation parameters, explaining the procedure to the patient and relatives, and taking due consent, the procedure was performed under ultrasound guidance with $10 \mathrm{ml}$ of $0.375 \%$ Ropivacaine which was deposited with the tip of the needle in the longus colli muscle after negative aspiration of blood. Spread of the drug was confirmed by bulging of the longus colli compartment and spread of drug on ultrasonography (USG). Horner's syndrome confirmed the success of the block. The patient showed an arrhythmia-free interval of $12 \mathrm{~h}$, but the monomorphic VT continued thereafter with a frequency of 6-7 episodes per day only amenable to synchronized cardioversion. He was intubated during one of these episodes and put on

Address for correspondence: Dr. Supriya D'souza, Department of Anaesthesiology, P D Hinduja Hospital and Medical Research Centre, Mumbai, Maharashtra, India. E-mail: supriyalynettedsouza@yahoo.com

This is an open access journal, and articles are distributed under the terms of the Creative Commons Attribution-NonCommercial-ShareAlike 4.0 License, which allows others to remix, tweak, and build upon the work non-commercially, as long as appropriate credit is given and the new creations are licensed under the identical terms.

For reprints contact: reprints@medknow.com

How to cite this article: D'souza S, Saksena S, Butani M. Calming the electrical storm: Use of stellate ganglion block and thoracic epidural in intractable ventricular tachycardia. Indian J Crit Care Med 2018;22:743-5. 


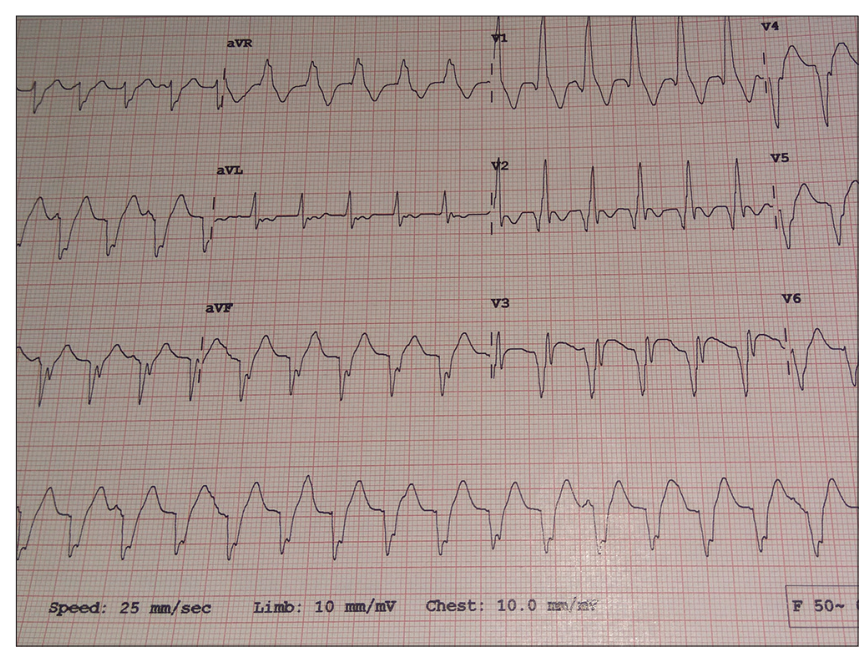

Figure 1: Monomorphic ventricular tachycardia during one episode

intermittent-positive pressure ventilation. Hypotension ensued and his right internal jugular vein and right radial artery were cannulated, and injection Noradrenaline was started. Sedation with Fentanyl and Midazolam infusion was started. Day 4 prompted a decision to try sympatholysis with a thoracic epidural. After due consent, under all aseptic precautions, in the left lateral position at the T2-T3 interspace, a 18G Tuohy needle was used and the epidural space was encountered with a paramedian approach at $4 \mathrm{~cm}$ using loss of resistance to saline method. The catheter was fixed at $8 \mathrm{~cm}$ at skin surface and secured with sterile dressing after tunneling. During the next episode of monomorphic VT, $5 \mathrm{ml}$ of $0.5 \%$ Lignocaine was injected with return to NSR within 3 min. After seeing this response, an infusion with $100 \mathrm{ml}$ of $1 \%$ Lignocaine with 100 mic of fentanyl was started at $5 \mathrm{ml} / \mathrm{h}$ with an elastomeric pump. After commencement of the infusion, there was a dramatic reduction in the occurrence of the VT with only one episode on day 5 which settled on bolus of $5 \mathrm{ml}$ of $0.5 \%$ lignocaine with 10 mic of Fentanyl through the epidural catheter. Plan was to do an electrophysiological (EP) study and catheter ablation of the arrhythmogenic pathway. Repeat TTE showed a LV clot of $2.3 \mathrm{~cm} \times 2 \mathrm{~cm}$ size. The EP study was deferred, and injection Heparin $5000 \mathrm{IU}$ bolus followed by $1000 \mathrm{IU} / \mathrm{h}$ infusion was started with regular monitoring of the activated partial thromboplastin time. The epidural catheter was kept in situ with only one episode of monomorphic VT on day 7 which settled on the abovementioned bolus. Relatives did not give consent for surgical left cardiac sympathetic denervation (LCSD). The patient suffered cardiac arrest with ventricular fibrillation on day 8 and succumbed.

\section{Discussion}

The autonomic nervous system plays a critical role in the genesis and maintenance of ventricular arrhythmias. ${ }^{[1]}$ An ES in ischemic heart disease usually manifests as recurrent ventricular arrhythmias that are resistant to antiarrhythmic drugs. ${ }^{[2]} \mathrm{VT}$ storm, defined as $>3$ episodes of VT within a 24 -h period, has high morbidity and mortality. Scar-mediated reentry due to a previous myocardial infarction (MI) is the most common cause, although reversible triggers include ischemia, decompensated heart failure, and electrolyte disturbances. VT storm is commonly managed with antiarrhythmic therapy, treatment of reversible causes (ischemia and electrolyte imbalance), and catheter ablation. ${ }^{[3]}$ The use of sympathetic blockade to stop VT storm is not novel as evidenced by the use of IV beta-blockers for treatment. Sympathetic tone can be reduced further by intubation and sedation, LSGB, TEA, or left cardiac sympathetic denervation (LCSD). We describe a case of VT storm we managed with SGB and TEA.

The left and right stellate ganglia provide the majority of the sympathetic innervation to the heart through postganglionic fibers, although the left stellate ganglion is quantitatively dominant at the ventricular level. ${ }^{[4]}$ MI may lead to partial denervation of these fibers at the level of the myocardium and paradoxically induce a supersensitivity to catecholamines, making the heart more vulnerable to the electrical induction of ventricular arrhythmias. ${ }^{[2]}$ Sympathetic denervation counteracts this pathologic process by reducing the amount of norepinephrine released at the ventricular level and increasing the ventricular fibrillatory threshold.$^{[4]}$ Coronary vasodilation and vagal stimulation to the heart are also increased. SGB also reduces the risk of cardiac arrhythmias by shortening the QTc interval. ${ }^{[5]}$ Landmark technique, USG, fluoroscopy, or computed tomography-guided techniques can be used. Nademanee et al. reported a prospective study of 49 patients with ES in which sympathetic blockade through beta-blocker or LSGB followed by oral Amiodarone was compared to Class I antiarrhythmic therapy. The 1-week survival for the sympathetic blockade group was $77.8 \%{ }^{[6]}$

TEA is a therapeutic option that selectively targets fibers that innervate the myocardium. ${ }^{[7]}$ Its immediate onset of action can interrupt the vicious cycle of recurrent shocks and may avert the need for intubation. TEA tends to have minimal effects on hemodynamic parameters including heart rate, mean arterial pressure, cardiac index, and central venous pressure. ${ }^{[8]}$

TEA with the potential of providing complete sympathetic blockade blocks segments C8-T4. While spinal segments $\mathrm{T} 1-\mathrm{T} 4$ give rise to the majority of cardiac accelerator fibers, C8 can form part of the inferior cardiac sympathetic nerve. Therefore, TEA, by inhibiting fibers that are proximal to both the left and right stellate ganglia, could be more effective than LCSD (which is a unilateral intervention). Other than Lignocaine, Bupivacaine, Levobupivacaine, and Ropivacaine with or without opioids can be used to achieve effective blockade. TEA can be instituted at the bedside without specialized equipment. TEA can also be used as a bridge to more definitive therapy. Application of local anesthetic to the epidural space results in almost immediate selective sympatholysis, which may be of particular benefit in patients with structural heart disease in whom the presence of scar may impede effective delivery of beta-blockade to the myocardial substrate. However, the dose of TEA is difficult to titrate as there are no markers that are easy to use other than an 
assessment of its efficacy in arrhythmia control. Bourke et al. reported a case study of 14 patients who underwent TEA, LCSD, or both, to control VT refractory to medical therapy and catheter ablation. After initiation of TEA, 6 out of 8 patients who had TEA had a large $(\geq 80 \%)$ decrease in VT burden. ${ }^{[9]}$

Other important treatment modalities which we could not use in our patient are implantable cardioverter defibrillator (ICD) and left cardiac sympathetic denervation. However, ICD implantation necessitates life-long and routine device replacement and is furthermore associated with device malfunction (including inappropriate shocks), infection, and psychological problems. ${ }^{[10]}$ LCSD is performed using either open or video-assisted thoracic surgery approach. Only left or bilateral denervation can be done. LCSD consists of resection and removal of the lower third to half of the stellate ganglia and T2-T4 thoracic ganglia as well as transection of the nerve of Kuntz (an intrathoracic nerve that connects the first and second thoracic nerves, bypassing the sympathetic chain between the T2 ganglion and stellate ganglion in some patients) when present. ${ }^{[1]}$ Vaseghi et al. conducted a study on 41 patients who underwent CSD (14 - left, 27 - bilateral), and there was a significant reduction in the burden of ICD shocks during follow-up compared to the 12 months before the procedure. Shock-free survival was greater in the bilateral group than in the left CSD group ${ }^{[11]}$ Horner's syndrome, pneumothorax, bleeding, adhesions/scarring, facial flushing, asymmetrical facial sweating, skin sensitivity, and neuropathic pain are known complications. ${ }^{[12]}$

\section{Conclusion}

This case highlights the need for awareness of alternative treatment modalities such as TEA and SGB which may be effective additions for the management of refractory ventricular arrhythmias, especially when other treatment modalities have failed and/or may serve as a bridge to more definitive therapy.

\section{Declaration of patient consent}

The authors certify that they have obtained all appropriate patient consent forms. In the form the patient(s) has/have given his/her/their consent for his/her/their images and other clinical information to be reported in the journal. The patients understand that their names and initials will not be published and due efforts will be made to conceal their identity, but anonymity cannot be guaranteed.

\section{Financial support and sponsorship}

Nil.

\section{Conflicts of interest}

There are no conflicts of interest.

\section{ReFERENCES}

1. Rubart M, Zipes DP. Mechanisms of sudden cardiac death. J Clin Invest 2005; 115:2305-15.

2. Zipes DP. Influence of myocardial ischemia and infarction on autonomic innervation of heart. Circulation 1990;82:1095-105.

3. Tung R, Shivkumar K. Neuraxial modulation for treatment of VT storm. J Biomed Res 2015;29:56-60.

4. Schwartz PJ. Cardiac sympathetic denervation to prevent life-threatening arrhythmias. Nat Rev Cardiol 2014;11:346-53.

5. Gadhinglajkar S, Sreedhar R, Unnikrishnan M, Namboodiri N. Electrical storm: Role of stellate ganglion blockade and anesthetic implications of left cardiac sympathetic denervation. Indian J Anaesth 2013;57:397-400.

6. Nademanee K, Taylor R, Bailey WE, Rieders DE, Kosar EM. Treating electrical storm: Sympathetic blockade versus advanced cardiac life support-guided therapy. Circulation 2000;102:742-7.

7. Eisenach JC, Tong CY. Site of hemodynamic effects of intrathecal alpha 2-adrenergic agonists. Anesthesiology 1991;74:766-71.

8. Hasenbos M, Liem TH, Kerkkamp H, Gielen M. The influence of high thoracic epidural analgesia on the cardiovascular system. Acta Anaesthesiol Belg 1988;39:49-54.

9. Bourke T, Vaseghi M, Michowitz Y, Sankhla V, Shah M, Swapna N, et al. Neuraxial modulation for refractory ventricular arrhythmias: Value of thoracic epidural anesthesia and surgical left cardiac sympathetic denervation. Circulation 2010;121:2255-62.

10. De Ferrari GM, Dusi V, Spazzolini C, Bos JM, Abrams DJ, Berul CI, et al. Clinical management of catecholaminergic polymorphic ventricular tachycardia: The role of left cardiac sympathetic denervation. Circulation 2015;131:2185-93.

11. Vaseghi M, Gima J, Kanaan C, Ajijola OA, Marmureanu A, Mahajan A, et al. Cardiac sympathetic denervation in patients with refractory ventricular arrhythmias or electrical storm: Intermediate and long-term follow-up. Heart Rhythm 2014;11:360-6.

12. Collura CA, Johnson JN, Moir C, Ackerman MJ. Left cardiac sympathetic denervation for the treatment of long QT syndrome and catecholaminergic polymorphic ventricular tachycardia using video-assisted thoracic surgery. Heart Rhythm 2009;6:752-9. 